\title{
BMJ open An exploratory qualitative interview study about collaboration between medicine and dentistry in relation to diabetes management
}

\author{
Susan M Bissett, ${ }^{1}$ Kerry Marie Stone, ${ }^{1}$ Tim Rapley, ${ }^{2}$ Philip M Preshaw ${ }^{1,3}$
}

To cite: Bissett SM, Stone KM, Rapley T, et al. An exploratory qualitative interview study about collaboration between medicine and dentistry in relation to diabetes management. BMJ Open 2013;3:e002192.

doi:10.1136/bmjopen-2012002192

- Prepublication history for this paper are available online. To view these files please visit the journal online (http://dx.doi.org/10.1136/ bmjopen-2012-002192).

Received 9 October 2012 Revised 17 January 2013 Accepted 21 January 2013

This final article is available for use under the terms of the Creative Commons Attribution Non-Commercial 2.0 Licence; see http://bmjopen.bmj.com

${ }^{1}$ School of Dental Sciences, Newcastle University, Newcastle upon Tyne, UK ${ }^{2}$ Institute of Health and Society, Newcastle University, Newcastle upon Tyne, UK

${ }^{3}$ Institute of Cellular Medicine, Newcastle University, Newcastle upon Tyne, UK

\section{Correspondence to}

Susan M Bissett:

s.m.bissett@ncl.ac.uk

\section{ABSTRACT}

Objective: To explore knowledge and attitudes regarding the links between diabetes and periodontitis of medical and dental healthcare professionals as well as those of people with diabetes.

Design: Qualitative interview study.

Participants: 4 people with diabetes, four dental professionals, three general practitioners (GPs) with a specialist interest in diabetes, one GP without a specialist interest in diabetes, three diabetic nurse specialists and two consultant diabetologists.

Setting: Primary and secondary care in Newcastle upon Tyne.

Methods: Interviews were audio recorded and transcribed verbatim. Framework analysis was used iteratively to achieve progressive focusing and to develop conceptual ideas.

Results: 3 inter-related themes emerged: (1) uncertain knowledge -a lack of familiarity about the links between periodontitis and diabetes; (2) unworkable knowledge - that even if the links between periodontitis and diabetes were known, it is impossible for anything to be done for patient benefit given the differing systems that medical and dental health professionals work in and (3) isolated knowledge - the perceived division that exists between the medical and dental professions has the potential to negatively impact on advances in diabetes patient care. The patients simply wanted all the healthcare professionals to be giving the same messages consistently and to help them access the (dental) care they need.

Conclusions: The research on the links between periodontal disease and diabetes appears to have limited impact on the organisation of diabetes care, and the divisions that exist between the medical and dental professions have the potential to negatively impact on patient care.

\section{INTRODUCTION}

Diabetes is a global public health problem, creating severe challenges for healthcare systems in many countries throughout the world. It is predicted that by the year 2025 ,

\section{ARTICLE SUMMARY}

Article focus

- Investigation into the knowledge and attitudes regarding the links between diabetes and periodontitis of medical and dental healthcare professionals as well as those of people with diabetes.

- Exploration of the reasons for limited collaboration between medical and dental healthcare teams in managing people with diabetes and periodontitis.

- Suggestions to improve awareness of the importance of oral health as part of diabetes management.

Key messages

- The evidence-base about the links between periodontal disease and diabetes appears to have limited impact on the organisation of diabetes care.

- The organisational and professional divisions that exist between the medical and dental professions have the potential to negatively impact on diabetes patient care.

- Local, regional and national initiatives could promote or coordinate the assessment and management of the oral health of patients with diabetes.

Strengths and limitations of this study

- This is an under-researched area and this phenomenon warrants further investigation with the aim to improve patient care.

- A small number of healthcare professionals and patients participated in this study conducted in the north east of England which may impact on transferability of the findings.

an estimated 380 million people will have type 2 diabetes. ${ }^{1}$ In the UK, 2.9 million people are diagnosed with diabetes and an estimated 850000 people have the condition but are currently undiagnosed. ${ }^{2}$ The North of England has the highest prevalence of both type 1 and type 2 diabetes among 
young people. ${ }^{3}$ Diabetes was initially identified to be a risk factor for periodontitis in the 1990s; the risk of periodontitis being increased by 2-3 times in a person with diabetes compared to individuals without it. ${ }^{4}$ The level of glycaemic control is key in determining the risk, ${ }^{5}$ and similar to the other complications of diabetes, the risk for periodontitis increases with poorer glycaemic control. ${ }^{67}$

Periodontal disease (gum disease) is very common and can range from gingivitis (reversible inflammation of the gingiva) to periodontitis (inflammation that extends from the gingiva to cause destruction of the surrounding tissues and alveolar bone resorption). ${ }^{8}$ Severe periodontitis, threatening tooth retention, affects approximately $10 \%$ of adults; while moderate periodontitis affects $40-60 \% .^{9}$ It is worthwhile to note that both conditions are generally painless and therefore can go unnoticed and untreated until the conditions reach an advanced stage. Meta-analyses confirm that reductions in glycated haemoglobin (HbAlc) can follow effective periodontal therapy. ${ }^{10}$ 11 Furthermore, in 2010 the Cochrane Collaboration published a review of studies that investigated the relationship between periodontal disease and the glycaemic control of people with diabetes. They reported a reduction in HbAlc of $0.40 \%$ 3-4 months after conventional periodontal therapy. ${ }^{12}$ The reason for this is still unclear but probably is because of reduced systemic inflammation, for example, reduced serum levels of tumour necrosis factor- $\alpha$ $(\mathrm{TNF}-\alpha)$ and interleukin 6 (IL-6). Reduction in HbAlc reduces the risk of diabetic complications. Research carried out by Stratton et al. in 2000 reported that each $1 \%$ reduction in $\mathrm{HbAlc}$ is associated with $21 \%$ reduced risk of any endpoint related to diabetes, $21 \%$ for deaths related to diabetes, $14 \%$ for myocardial infarction and $37 \%$ for microvascular complications. ${ }^{13}$

Education plays a significant role in the management of diabetes. A team of healthcare professionals provide advice to people with diabetes regarding control of their blood glucose, and specialist monitoring of their eyes, feet, heart and kidneys. The addition of dental monitoring could enable people with diabetes to become informed of the potential periodontal complications of their disease. However, the recent Adult Dental Health Survey (2009) stated that $39 \%$ of the population do not attend a dentist. ${ }^{9}$ The main reason for dental nonattendance (after 'having nothing wrong') is not being able to find an NHS dentist.

Our perception was that healthcare professionals (medical and dental) and people with diabetes have little-to-no knowledge of the evidence linking periodontal disease and diabetes and therefore are not routinely informing their patients about the importance of regular dental monitoring. To the best of our knowledge, no research has been carried out in this area before and our aim was to investigate this perception further. Qualitative research is ideally suited to an initial exploration of a phenomenon such as this, ${ }^{14}$ and conducting interviews with a range of healthcare workers and people with diabetes enabled us to begin to map their knowledge of the links between periodontitis and diabetes.

\section{METHODS}

Prior to the start of the research, ethical approval was obtained from the County Durham and Tees Valley NHS Research Ethics Committee (Ref 10/H0908/64). We carried out semistructured interviews with 17 purposively sampled participants to cover a range of healthcare professionals and patients: four people with diabetes, four dental professionals, three general practitioners (GPs) with a specialist interest in diabetes, one GP without a specialist interest in diabetes, three diabetes nurse specialists and two consultant diabetologists. The people with diabetes were selected from a database of individuals who have previously participated in research within our department and had expressed an interest in participating in future projects. Participants from the dental and healthcare professional cohorts were selected via snowballing sampling, ${ }^{15}$ initially contacting professionals already known to the researchers via letter or email invitation, and as data analysis progressed, our emergent findings suggested that we needed to expand the types of health professionals we sampled. We also sought to sample for how knowledge was distributed across a range of key stakeholders involved in diabetes management, as we wanted to begin to describe and map the diversity across groups. Participants were either invited to the School of Dental Sciences at Newcastle University for the interview or the researchers offered to visit the participant's place of work, provided a private room could be secured.

\section{Data collection and analysis}

Interviews were recorded and transcribed verbatim. A conversational style of interviewing was adopted, to encourage a comfortable and fluent dialogue which was rich in detail, while using an interview schedule as a reference to ensure that all key topics were covered. Framework analysis was utilised as the interviews started with an a priori set of questions relating to knowledge and issues surrounding integration/application within a primary and secondary healthcare setting. ${ }^{16}$ Transcripts were analysed in an iterative cycle, that is, analysis was carried out concurrently with the interview process which allowed new lines of enquiry to be followed up in subsequent interviews. Initial analysis involved familiarisation with the interviews: listening to the audio tapes alongside active reading of the transcripts, making notes and highlighting issues of interest. Identification of these issues enabled a thematic framework to be created. These initial themes had arisen from the study objectives and a priori issues, but formed a foundational framework which was later adapted as the on-going data analysis provided new themes. Data were then indexed and charted within 
thematic frameworks. Finally, the data and themes were reviewed and refined, until associations and explanations could be drawn from the definitive concepts.

\section{RESULTS}

Four people with diabetes and 13 healthcare professionals participated in this research. The participants with diabetes had a mean age of 47 years (range 3062 years), three had type 2 diabetes, three were male, and the mean time since diagnosis of diabetes was 16.5 years (range 4-22 years). The healthcare professionals included seven males and six females, who had achieved their primary qualifications 8-37 years previously. Those who specialised in diabetes management (the GPs with a special interest in diabetes, the diabetes nurse specialists and the diabetes consultants), had been working in the specialty for 2-36 years. Interviews lasted between 30 and $60 \mathrm{~min}$, and were conducted from March to September 2011.

Three inter-related themes emerged: (1) uncertain knowledge-a lack of familiarity about the subject area; (2) unworkable knowledge-that even if the links between periodontitis and diabetes were known, it is impossible for anything to be done for patient benefit given the differing systems that medical and dental healthcare professionals work in and (3) isolated knowledge-the perceived division that exists between the medical and dental professions has the potential to negatively impact on advances in diabetes patient care.

Uncertain knowledge: If the interviewees were not already aware of the links between periodontitis and diabetes, this concept was introduced in the study Information Sheet, and the topic was then opened up for discussion during the interview. Three participants (two diabetes nurse specialists and one patient) claimed to have never heard about the links between diabetes and periodontitis prior to reading the Information Sheet (box 1(1)). Generally, the healthcare professionals reported having a minimal knowledge-base on this topic. The links did make sense to the interviewees in the context of what they already knew about the complications of diabetes in general and the effect of infection and inflammation on blood glucose levels. The dental professionals were all familiar with the research linking the diseases. During the course of the interviews, the large body of research that has been carried out in this area, including the recent Cochrane collaboration systematic review, was discussed. Several of the healthcare professionals reacted with surprise to this information, as they had been unaware of the scope of the evidence-base (box 1(2)). There was generally a request for more information on this topic (box 1(3)). Two healthcare professionals did note that they would need more evidence before they would consider the relevance of the research for their own clinical practice (box 1(4)). Patients with diabetes were also poorly informed about the links between diabetes and periodontitis (box 1(5)).

\section{Box 1 Uncertain knowledge}

1. "No, I can't remember anything umm that I've been-I mean you know, when you've worked in diabetes for a long time, that part of the education, we would obviously-high glucose levels as we know can affect all parts of the body because its affecting cells, mm, so in lack of-poor diet, which we know that that's an area where they can have potential problemsbut there's not sort of a module-there's not a section where we would separately cover dental" (HCP04, diabetes nurse specialist)

2. “I suspect it's not widely known and I hadn't heard about that Cochrane Review and I suspect nobody else has heard about the Cochrane Review. To be quite honest l'm surprised there was enough evidence for the Cochrane guys to actually do a review.....because I wasn't aware that there was all that much evidence...0h, 690 papers, seven studies, they ended up with, which sounds awful, but it's actually not bad for a formal, umm- yeah" (HCP06, consultant diabetologist)

3. "Uhh-I might have to get you to tell me the links. I mean my main issue is probably picked up as just linking infections [in diabetes] but I don't know if there's something else that I should know about that I don't know about" (HCP03, GP with specialist interest in diabetes)

4. "And the question is how- would that make any difference? If somebody wants to take care of their teeth, they tend to be already under [the care of] a dentist [or] hygienist, and those who don't want to, well is it going to- is there any evidence that a doctor telling them to go and see their dentist, because the $X, Y$ and $Z$, is actually going to make any real difference to their behaviour? If there is, then obviously, then we should take that on board, but I'm not aware of anything that would say that we should." (HCP02, GP with specialist interest in diabetes)

5. “No, I don't think that I was [told about the links between diabetes and periodontitis]. I think the first I had heard of it was when I went to the previous dentist and she said I had [advanced] gum disease." (DP04, patient with diabetes)

Unworkable knowledge: In broad terms, the interviewees felt that consideration of the oral health status of people with diabetes was a good idea, and they could appreciate the benefits of reduced oral inflammation in terms of having a positive impact on blood glucose levels. However, the absence of any local, regional or national structures to promote or coordinate the assessment and/ or management of the oral health of patients with diabetes was felt to be a central source of the problem (box 2(1)). It is not that the impact of oral health on diabetes has never been considered in national guidelines. One of the interviewees had considerable experience in developing and implementing guidelines in diabetes care, and identified that the limited resources for managing diabetes means that there are many priorities competing for inclusion within the guidance given to healthcare professionals (box 2(2)). That interviewee highlighted the potential of commercial funding to raise the profile of oral health within the context of diabetes at the national public policy level which could in turn 


\section{Box 2 Unworkable knowledge}

1. "It's a very interesting question because we don't do very much of it. Umm- what we do is not necessarily what I think we should be doing, umm, what we do is, umm, opportunistically watch for situations in which we might think there is an issue over oral health... Umm-so I mean we look out for people who've got bad teeth, periodontitis, and you know, think it might be interfering with blood glucose control or something like that ...Umm, what we perhaps don't do is, is do that in any structured fashion." (HCPO7, consultant diabetologist)

2. "Yeah, I mean-there are two issues there, one is we've considered putting diabetes and oral health into the NICE National Guidelines, umm, what actually caused it to drop out was priorities...Because NICE had only limited funding...And we dropped-I can't- don't ask me what else it was, but we dropped a series of topics on the basis that we didn't have the funding, enough or the time to cover them...Umm, so, sodental management, teeth management and things, didn't get into the NICE Guidelines. Now with the IDF, International Diabetes Federation Guidelines, the opposite happened in a sense, umm, in that surprise, surprise, it was Colgate who came in and offered money to the IDF...So it comes down, as it always is, comes down to funding, but funding relates to priorities. I mean you will always do your first priority with the minimum amount of money...Y You need a lot more to go into other things, and you know, let's be honest about it, dental health has not got a lot of exposure in the diabetes world in any of the time l've been involved." (HCP07, consultant diabetologist)

3. "...that's one of the reasons, yeah, it's not in the template, so that's why we don't ask" (HCP08, GP without a specialist interest in diabetes)

4. “...it's just partly through to training, partly throughsometimes through to time pressures, and the way we work, and also just a general, sometimes you know, I want to be my own person type thing, and a sort of willing to accept, whereas the nurses tend to be quite happy. They [diabetic nurse specialists] prefer working behind protocols, having a system..." (HCP02, GP with a specialist interest))

influence local practice. A common perception among the interviewees was that without the inclusion of oral health assessment into the everyday organisation of diabetes care, for example, through the electronic template that supports the management of their diabetic patients, then research into the impact of periodontitis and its treatment on glycaemic control is never likely to have an impact on patient care. They indicated the potential of the template to 'shape' aspects of patient management (box 2(3)) especially in relation to diabetic nurse specialists, who were often positioned as actively employing more protocol-driven care (box 2(4)).

Isolated knowledge: Healthcare professionals alluded to a feeling of isolation when dealing with the oral problems of their patients. A common theme from the medically qualified interviewees was that they felt there is little education in dental subjects at the undergraduate (medical student) level and this seemed to impact on their considerations about the systemic impact of oral diseases. It was accepted that the medical curriculum was unable to cover everything and that the mouth is generally considered by medics as being the 'realm of dentists'. However, their training failed to prepare them for managing the dental complaints that they faced during their clinical practice. The problems that can arise as a result of their limited knowledge of dentistry were highlighted by the GPs who reported the frequent occurrence of patients coming to visit them when they had an oral or dental problem. The existence of a separate profession who specialise in dentistry will not prevent the public from going to their doctor if they have a problem with their mouth (rather than going to a dentist); and doctors recognised, albeit reluctantly at times, the need to provide at least some limited care (box 3(1)). This reluctance was tangible and the issue of a division between the medical and dental professions arose repeatedly (box 3(2)). GPs felt a strong sense of isolation when put in the position of trying to manage patients with dental problems. One interviewee stated that he felt '....untrained and unsupported...' (HCP02, GP with a specialist interest in diabetes) in this area. GPs do not like having to see patients with 'jaw ache' who cannot, for whatever reason, see their dentist. GPs also seemed to question the contribution of dentists to the care of all patients at all times, with a perception that dental practices were run as small businesses, somewhat outside the NHS (box 3(3)). In turn, dentists were also aware of a 'divide' between themselves and their medical colleagues, and perceived the negativity that they detected as doctors asserting their superiority (box $3(4))$. The patients indicated that they felt their GPs had a pivotal role in informing them about all the possible complications of diabetes and that better collaboration between medical and dental healthcare providers could only be of benefit (box 3(5)).

All three factors meant that patients appeared to have a poor level of access to timely, good-quality knowledge. The patients with diabetes reported that they would prefer to be informed about their increased susceptibility to developing periodontitis. They acknowledged that some people with diabetes are dismissive about the disease, '...if they don't feel ill with it [diabetes] it'll go away' (DP04); and that it takes a long time to understand the full implications of having diabetes, '...I'm still trying to get a handle on what diabetes actually is' (DP03). However, while the healthcare workers struggled with how to put oral health initiatives into practice within the systems they work in, the patients simply wanted all the healthcare professionals to be giving the same consistent messages and to help them access the (dental) care they need.

\section{DISCUSSION}

This research was undertaken to explore the knowledge of both people with diabetes and healthcare 


\section{Box 3 Isolated knowledge}

1. "...I think that the pressure on the medical schools to incorporate, but again that's going to be hard work because there are going to be lots of pressures on there, umm, but I think a greater focus on oral health will probably make a difference, at least in terms of dealing with common problems and how to access help and all the rest of it...you have to provide some limited care, umm, how effective and good we are at providing that care, that's probably debatable but, you know, uh, we have to do what we can..." (HCP02, GP with a specialist interest in diabetes)

2. "Barrier's probably too grand a title for it, umm it's a combination of time pressures and it's not my responsibility. You know, so it's not- and when I say it's not my responsibility, you know, that sounds like a demarcation dispute, but l'm not, there's so many other things l've got to deal with, with people with diabetes, that, well, that's really the dentist's job so l'll not do it." (HCP01, GP with a specialist interest in diabetes)

3. “...I don't think many NHS dentists that l've come across understand- they don't seem to understand that they're part of a National Health Service, in effect, they see themselves as isolated little businesses, that are being run in their own way, and for a lot of them, not having access to emergency care, they seem to think is completely acceptable. We don't and the patients don't really either, but the dentist seems to think it's quite reasonable for somebody with a painful jaw to wait 10 days. You wouldn't accept that in general medical practice. I don't think you should accept it in general dental practice." (HCP01, GP with a specialist interest in diabetes)

4. "... I think some of our medical colleagues look down on their dental colleagues as being something inferior, and therefore they're not going into dentistry. That's probably a harsh generalisation but sometimes I think that's true. It's, you're a dentist what do you know...uh, medical colleagues tend not to think of the mouth as being of any importance whatsoever and that's the realm of dentists." (DEN03, dental professional)

5. "...I don't think she [my GP] has asked me if l'm registered with a dentist, but we do have lots of discussions about lots of things... if there was a push on GPs to advise patients about [the importance of oral health], she would tell me-if she'd received the information. If she gets anything new she tells me immediately. We have an extremely good relationship. It's mostly conversation... she doesn't bully me into anything... but we sort of like come to conclusions..." (DP03, patient with diabetes)

professionals who manage people with diabetes regarding the links between periodontitis and diabetes. Interviews were conducted with a broad range of key stakeholders in a diabetes patient care pathway and even though the numbers within each relevant group were relatively small, a consistent message appeared across the groups. Despite the fact that there is a substantial body of research evidence linking these two diseases (including a Cochrane systematic review), ${ }^{12}$ this information does not seem to have impacted on these healthcare professionals, including those who specialise in managing the care of people with diabetes. The World
Health Organisation has identified that oral health is integral to general health and is essential for general well-being. ${ }^{17}$ Furthermore, periodontal disease is highly prevalent $^{8}$ and impacts on many aspects of daily living, affecting confidence, social interactions and food choices. ${ }^{18}$ The patients with diabetes whom we interviewed expressed a desire to be informed about their increased susceptibility to periodontitis and the potential benefits in terms of improvements in glycaemic control (reductions in HbA1c) that might occur following the treatment of periodontal disease. The healthcare professionals' accounts suggested that they were relatively inactive, however, when questioned about their role in this area of diabetes management.

Healthcare professionals' knowledge about the links between diabetes and periodontitis was found to be relatively minimal in this study population. Further research is necessary to identify how these findings apply more broadly; for example, a questionnaire survey could target healthcare professionals across a range of disciplines and at various geographical locations. Although the benefits of screening for, and treating, periodontitis in patients with diabetes were recognised, a number of barriers were identified that would need to be addressed before patients would benefit from the evidence-base. Clearly, awareness of the current evidence-base could be raised in a range of settings, including undergraduate, postgraduate and continuing medical education. As clinical routines are based on a mixture of education, evidence, experience and habit, improving the awareness of healthcare professionals of the bidirectional relationship between periodontitis and diabetes alone, ${ }^{7}$ will not necessarily translate to patient benefit. The inclusion of the oral complications of diabetes into local care pathways, electronic templates and national diabetes guidelines could also help. However, protocols and guidelines, although an excellent way to implement new clinical interventions, are not the only way to influence the practice of healthcare professionals. ${ }^{19}$ The role of key opinion leaders, commercially supported local networking events and peer-led discussion forums such as lunchtime practice seminars, were also highlighted by the interviewees as being potentially effective ways to raise general awareness of the links between periodontitis and diabetes, and the current evidence-base such as the Cochrane review.

Any implementation of change needs to be situated with the context of both public and professional cultures in relation to oral health. The last Adult Dental Health Survey (2009) stated that the most frequent reasons for dental non-attendance are that patients cannot find an NHS dentist (or their dentist has just changed from providing NHS care to private care only), they cannot afford to go, or they are afraid to go to the dentist. ${ }^{9}$ In the UK, the relationship between a patient and their dentist is different from the relationship with their doctor, because even when receiving NHS dental treatment, there is a financial element involved with virtually 
every appointment (unless the patient is in receipt of certain benefits). ${ }^{20}$ As yet, we have relatively little knowledge of how people with diabetes seek oral healthcare and how this could impact on promoting oral health screening. In this research, we have also documented some of the divisions that exist between the medical and dental professions, specifically related to the links between periodontitis and diabetes. These divisions are not new; they have existed since the medical and dental professions first emerged as independently regulated professions which, in the case of Britain, was in the early to mid-20th century. ${ }^{21} 22$ From the outset, the distinction was made that dental health was the jurisdiction of dentists and quite separate, although closely related, to general health. This state of affairs has existed with little dispute from either professions for many years. Clearly, this tension has the potential to impact on any intervention introduced to promote interprofessional collaboration.

As the prevalence of diabetes is growing, so is the cost of treating it. During 2010/2011, the direct cost to the NHS for treating diabetes was $£ 9.8$ billion (the indirect cost being $£ 13.9$ billion). ${ }^{23}$ In total, $80 \%$ of the direct costs were reported to go towards treating the complications of the disease, many of which are preventable. Diabetes UK reports that the economic burden to the NHS, for both direct and indirect costs of diabetes is estimated to rise to $£ 16.8$ billion by $2035 / 2036$, which equates to a predicted $17 \%$ of the total health resource expenditure for the NHS. ${ }^{23}$ Diabetes is a major risk factor for periodontitis ${ }^{4}$ and periodontitis appears to impair glycaemic control in people with diabetes. ${ }^{24}$ Furthermore, periodontal treatment results in improvements in glycaemic control, which although modest (HbA1c reductions of approximately $0.4 \%$ ), could be clinically relevant, and are similar to reductions achieved by some oral therapies such as the gliptins. Interprofessional collaboration in the form of education and practice may enhance the management of chronic diseases, such as diabetes, through the management of their common risk factors, underlying social determinants and their common biological pathways. $^{25}$ Interprofessional collaboration already exists for management of certain complications of diabetes, such as the established screening programmes to assess for retinopathy.

Clearly, the value placed on screening programmes will be linked to the morbidity associated with the complication that is being assessed. Given that patients and most healthcare providers in our sample appeared to be relatively uninformed about the links between diabetes and periodontitis, assessing oral health may be regarded as having a lower priority than assessing retinal health, for example. On the other hand, periodontitis has a major impact on quality of life, ${ }^{18}$ and the early loss of teeth can be a very distressing outcome for any patient. Therefore, diabetes care teams could inform patients with diabetes about their increased risk for periodontitis, and of the importance of obtaining dental care, as
Box 4 Suggested brief oral health intervention for diabetes care providers to implement with their patients with diabetes

ASK if the patient attends the dentist.

INFORM them of their increased susceptibility to periodontitis. If the patient has periodontitis, inform them about the potential benefits of getting their periodontitis treated; in the form of improved glycaemic control (reductions in $\mathrm{HbA} 1 \mathrm{c}$ ).

- ADVISE them to seek professional dental assessment and regular oral screening.

proposed in the suggested brief oral health intervention (in box 4). The validity of such an approach would need to be tested in future research. It may also be worthwhile investigating the use of leaflets or posters to provide more information for patients, and ultimately, the inclusion of a dental hygienist into diabetes care teams to undertake oral health assessments could benefit patients and enhance interprofessional working.

The small sample numbers in this study is a limitation, although statistical representation is rarely sought in qualitative research. ${ }^{26}$ By focusing on how knowledge was distributed across a range of different stakeholders we only recruited a small number of people within each category, so we were unable to further document the diversity of knowledge within specific groups. In relation to patients, there is a clear need for further in-depth research involving a broad range of patients, with both type 1 and type 2 diabetes, to explore their experiences of oral health in the context of their diabetes. This was an exploratory study in a very under-researched area in which we sought to scope the variance in the phenomenon, while accepting the potential for bias in this relatively small sample. Despite these limitations, the same key issues consistently emerged across the sample, and we feel they offer a useful direction for further research.

\section{CONCLUSION}

A substantial body of evidence exists linking diabetes and periodontitis; however, it appears to have had a limited impact on the work of health professionals. If people with diabetes are unable to benefit from the findings of over 20 years of research, including a Cochrane review, practical solutions need to be found. Education is potentially needed but, without other measures aimed at the organisation of care and ameliorating professional tensions it will not translate into patient benefit. This exploratory study identified a potential need for professional collaboration to develop an oral health educational initiative that would enable healthcare professionals to deliver clear and effective advice to people with diabetes regarding the importance of good oral health as part of their overall diabetes care. Clearly, further research is needed to help clarify the extent of this issue and to establish effective ways to implement change. 
Acknowledgements We thank the participants who generously gave their time.

Contributors SMB, KMS, PMP and TR designed the study. SMB and KMS recruited the participants and carried out the interviews. SMB, KMS identified the thematic framework for analysis. SMB, KMS, PMP and TR charted, refined and interpreted the data. SMB wrote the paper and all authors revised it. SMB is a guarantor. All authors read and approved the final manuscript.

Funding SMB and KMS were funded by The Oral and Dental Research Trust in partnership with Colgate to undertake this study. This funding was awarded in an open competition as part of the DCP Colgate research awards (DCP, Dental Care Professional), administered by the Oral and Dental Research Trust.

Competing interests None.

Ethics approval County Durham and Tees Valley Proportionate Review Service; Ref 10/H0908/64.

Provenance and peer review Not commissioned; externally peer reviewed.

Data sharing statement No additional data are available.

\section{REFERENCES}

1. van Dieren S, Beulens JWJ, van der Schouw YT, et al. The global burden of diabetes and its complications: an emerging pandemic. Eur J Cardiov Prev R 2010;17:s3-8.

2. Diabetes UK. Guide to diabetes/what type of diabetes do I have. http://www.diabetes.org.uk/Guide-to-diabetes/ What-type-of-diabetes-do-I-have/ (accessed 17 Jan 2013).

3. Haines L, Kramer Z. Growing up with diabetes: children and young people with diabetes in England. London: Royal College of Paediatrics and Child Health, 2009.

4. Mealey BL, Ocampo GL. Diabetes mellitus and periodontal disease. Periodontol 2000 2007;44:127-53.

5. Tsai C, Hayes C, Taylor GW. Glycemic control of type 2 diabetes and severe periodontal disease in the US adult population. Community Dent Oral Epidemiol 2002;30:182-92.

6. Pihlstrom BL, Michalowicz BS, Johnson NW. Periodontal diseases. Lancet 2005;366:1809-20.

7. Soskolne WA, Klinger A. The relationship between periodontal diseases and diabetes: an overview. Ann Periodontol 2001, 6:91-8.

8. Preshaw PM, Alba AL, Herrera D, et al. Periodontitis and diabetes: a two-way relationship. Diabetologia 2012;55:21-31.

9. Morris J, Chenery V, Douglas G, et al. Service considerations-a report from the Adult Dental Health Survey 2009. In: O'Sullivan I. ed.
Adult Dental Health Survey 2009. London: NHS Information Centre for Health and Social Care, 2011:1-19.

10. Janket SJ, Wightman A, Baird AE, et al. Does periodontal treatment improve glycemic control in diabetic patients? A meta-analysis of intervention studies. J Dent Res 2005;84:1154-9.

11. Darré L, Vergnes JN, Gourdy P, et al. Efficacy of periodontal treatment on glycaemic control in diabetic patients: a meta-analysis of interventional studies. Diabetes Metab 2008;34:497-506.

12. Simpson TC, Needleman I, Wild SH, et al. Treatment of periodontal disease for glycaemic control in people with diabetes. Cochrane Database Syst Rev 2010;5:CD004714.

13. Stratton IM, Adler Al, Neil HA, et al. Association of glycaemia with macrovascular and microvascular complications of type 2 diabetes (UKPDS 35): prospective observational study. BMJ 2000;321:405-12.

14. Pope C, Mays N. Reaching the parts other methods cannot reach: an introduction to qualitative methods in health and health services research. BMJ 1995;311:42-5.

15. Patton MQ. Qualitative evaluation and research methods. Thousand Oaks, CA: Sage, 2002

16. Ritchie J, Spencer L. Qualitative data analysis for applied policy research. In: Bryman A, Burgess RG. eds Analysing qualitative data. London,New York: Routledge, 1994:172-94.

17. Petersen PE. The World Oral Health Report 2003: continuous improvement of oral health in the 21st century-the approach of the WHO Global Oral Health Programme. Community Dent Oral Epidemiol 2003;31:3-24.

18. O'Dowd LK, Durham J, McCracken GI, et al. Patients' experiences of the impact of periodontal disease. $J$ Clin Periodontol 2010;37:334-9.

19. Gabbay J, le May A. Evidence based guidelines or collectively constructed "mindlines?" Ethnographic study of knowledge management in primary care. BMJ 2004;329:1013.

20. Exley C. Bridging a gap: the (lack of a) sociology of oral health and healthcare. Sociol Health III 2009;31:1093-108.

21. Adams T. Dentistry and medical dominance. Soc Sci Med 1999;48:407-20.

22. Davis P. The social context of dentistry. London: Croom Helm, 1980.

23. Hex N, Bartlett C, Wright $D$, et al. Estimating the current and future costs of type 1 and type 2 diabetes in the UK, including direct health costs and indirect societal and productivity costs. Diabetic Med 2012;29:855-62.

24. Taylor GW, Burt BA, Becker MP, et al. Severe periodontitis and risk for poor glycemic control in patients with non-insulin dependent diabetes mellitus. J Periodontol 1996;67:1085-93.

25. Sheiham A, Watt RG. The common risk factor approach: a rational basis for promoting oral health. Community Dent Oral Epidemiol 2000;28:399-406.

26. Pope $\mathrm{C}$, Mays N. Critical reflections on the rise of qualitative research. BMJ 2009;339:b3425. 